\title{
Real-time Measuring and Monitoring of Relevant Parameters in Complex Chain Conveyor Systems
}

\author{
Jens Strobel*, Jens Sumpf, Ralf Bartsch, Markus Golder \\ Professorship of Conveying Engineering and Materials Handling, Chemnitz University of Technology, Germany \\ * Correspondence:jens.strobel@mb.tu-chemnitz.de
}

Received 27 October 2020; Accepted 28 October 2020; Available online 7 December 2020

(C) 2020 by Strobel et al. This is an open access article distributed under the Creative Commons Attribution License (CC-BY 4.O), which permits unrestricted use, distribution, and reproduction in any medium, provided the original work is properly cited.

\begin{abstract}
In continuous conveyor systems with circulating traction mechanisms, e.g. chains, the kinematicdynamic movement behaviour of the traction mechanism is of great importance. Force and vibration analyses can be used to detect overloads and other irregularities in the conveying process. This helps to prevent damage that can lead to a complete failure of the conveyor system. Such analyses are particularly interesting for slide chain conveyors with plastic chains, as these react very sensitively to system overload due to limited thermal and mechanical characteristics as well as often a complex track layout. This article shows how accelerations and tractive forces can be continuously recorded, transmitted and analysed by sensors integrated into the chain links. In addition to the discussion of test results, potential applications of the measuring chain are shown.
\end{abstract}

KEYWORDS continuous conveyor, slide chain, tractive force, strain gauges, inertial measuring unit

\section{Introduction}

Chain conveyor systems are often used to realise material flow in many branches of industry. They are used for the internal transport of packaged goods and cover a wide range of applications due to their different chain types and sizes as well as their versatile adaptable chain top face.

Conveyors with plastic chains are becoming increasingly important. The used thermoplastics enable an economical production as well as an energy-efficient and lubrication-free operation. Their adaptation to the logistic processes is easy to perform due to a commonly modular structure of racks, drives, redirections, wheeled curves, sliding curves and guiding profiles (see Figure 1).

The highest possible reliability and service life are relevant for conveyor systems, which require a maximum load adapted to the overall system. Unexpected failure of the conveyor system must be avoided at all costs, as in most cases this leads to considerable disruptions in the production process. This applies in particular to plastic chains, as these have limited 

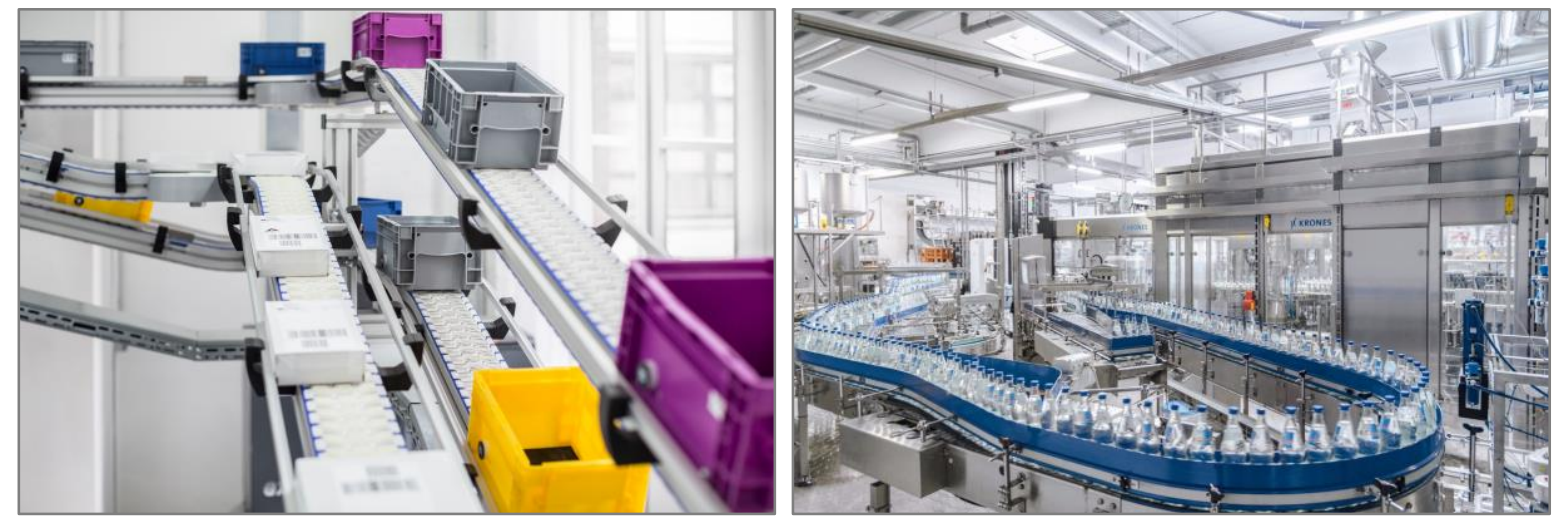

Figure 1: Complex sliding chain conveyor systems; left: Bosch Rexroth [16], right: Krones AG [17]

strength compared to classic steel chains, combined with a high temperature sensitivity, e.g. due to frictional heat. Therefore, an overload can cause chain breaks as well as a strong heating with excessive wear.

In order to prevent this and to detect and react to irregularities at an early stage, it is very important to know the exact loads within the chain system during operation.

\section{Initial Situation and Problem}

In conveyor systems a chain link is subject to a cycling traction load, which is shown schematically in Figure 2. Starting at the outlet from the drive, where the chain is suspended in a so-called chain bag and is load-free, it passes through the lower guiding rail up to the redirection. Afterwards the sliding chain is available in the upper area for the transport of goods. The driving force is counteracted by various frictional forces, which are generated

- between the guiding rails and the chain,

- between the goods to be conveyed and the chain during accumulation,

- in chain joints and

- in bearings.

The chain tractive force $F_{Z}$ therefore increases over the entire circulation and reaches its maximum value $F_{Z \max }$ at the entry into the drive sprocket, which in the case of non-tractioned chains corresponds to the circumferential force $F_{U}$ used for designing drives.

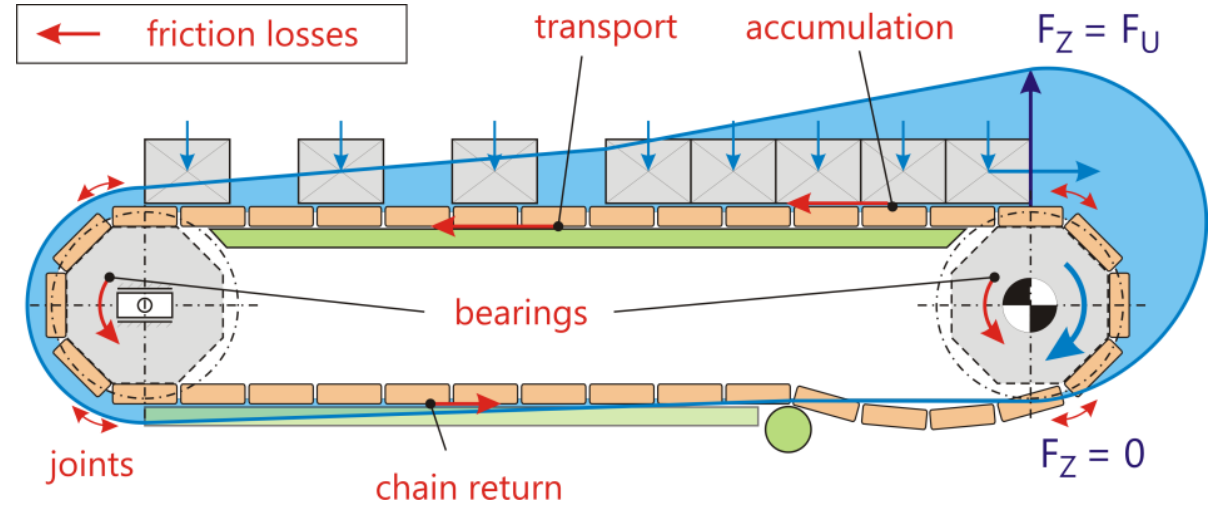

Figure 2: Curve of the chain tractive force in the conveyor system 
The most common types of failure in sliding chain conveyor systems are

- wear at the friction points, especially in the chain joints,

- thermal overload of chains and/or sliding rails as well as

- chain breakage, which immediately brings the system to a standstill.

These failures are mainly caused by an excessive chain tractive force. The maximum permissible chain tractive force $F_{Z \max }$ in operating condition, i.e. with pulsating traction load, is provided by the manufacturer. The specification of this force varies greatly from a simple limit value, which is about $25-30 \%$ of the breaking strength, to the determination in fatigue strength diagrams and the consideration of certain process variables such as speed, start-up frequency or ambient temperature. [1-7]

The calculation of the chain tractive force $F_{Z}$, even for complex conveyor systems with a given layout and load, is state of the art. It can be performed in an application-oriented manner using simplified methods, e.g. [1] or for more precise analysis by means of detailed calculation approaches [9-11]. All these calculations base on some practical simplifications, e.g. the neglect of longitudinal and joint friction. The required coefficients of friction are known to depend very much on the load and ambient conditions such as surface pressure, relative speed or temperature and can therefore not be considered as constant during the chain circulation. [12-14]

Another shortcoming of current calculations is the fact that they assume a quasi-static increase in tractive force during chain circulation, because the periodic threshold load is very slow. During the circulation, however, the chain links are additionally stressed by start-ups under load or isolated impacts between chains and the guide system. It is also known that external influences such as the polygon effect at the drive, the loading and unloading with goods, the track layout as well as the mechanical chain properties can cause a sliding chain conveyor system to oscillate. This results in a time-varying tractive force in the chain, the maximum values of which can significantly exceed the statically calculated tractive force [15]. These dynamic load peaks can also contribute to a considerable extent to the wear of the sliding chains and thus to the reduction of the service life.

\section{Objective}

As the highest tractive force occurs when the chain enters the drive, it can be determined relatively easily by measuring the drive torque (e.g. via a torque measuring shaft or roughly estimated from the data of the drive unit [8]) and the pitch circle radius of the drive sprocket. However, the disadvantage of this variant is that only the cumulative friction losses of the entire system can be gathered.

For more precise analyses, it is therefore desirable to measure directly in the circulating chain. In this way, the development of the tractive force in the chain and vibrations within the conveyor can be observed separately and discontinuities can be detected precisely in 
their positions. The objective is therefore the integration of a measuring unit directly in the chain. For use in real conveyor systems, the following specific requirements must be met:

- The integration of the required measuring electronics must be carried out without changing the outer geometry of the chain in order to ensure the movability of the chain and unhindered transport of goods.

- The entire measuring unit must be effectively protected against external influences and should at least have the durability of the conveyor chain in continuous operation for industrial use. This should prevent a new weak point in the conveyor system.

- The amount of modifications to the conveyor system should be kept to a minimum.

- The measured values should be recorded continuously during the complete chain circulation and at a sufficiently high sampling rate.

- The readout of the data should be contactless by radio and if possible, in real-time.

- The energy supply of the measuring system must be as contactless as possible or, if moving along energy storage devices are used, a sufficiently long measuring time as well as comfortable exchange or recharging must be ensured.

\section{Realisation of a Measuring Chain Section}

Taking into account the above-mentioned requirements, available hardware solutions were sought on the market, although most standard devices were not suitable due to measuring or geometric limitations. In the end, a prefabricated board with the dimensions $17 \times 46 \mathrm{~mm}$ was used. This has a 32 bit microcontroller, a Wi-Fi module and an inertial measurement unit (IMU) with 6 axes. The connection of the strain gauge was realised by a second board. On this board there is a circuit for interrogation of load cells or strain gauges. This has a signal amplifier and a 24 bit analogue-digital converter and is designed in such a way that a full measuring bridge can be connected directly.

In the first phase of development, the measuring electronics and the periphery were roughly adapted. In particular, the power supply used requires attachment to several links, which are connected to form a measuring chain section (see Figure 3). This is inserted into the chain strand of the conveyor and can directly measure the forces and accelerations occurring there.

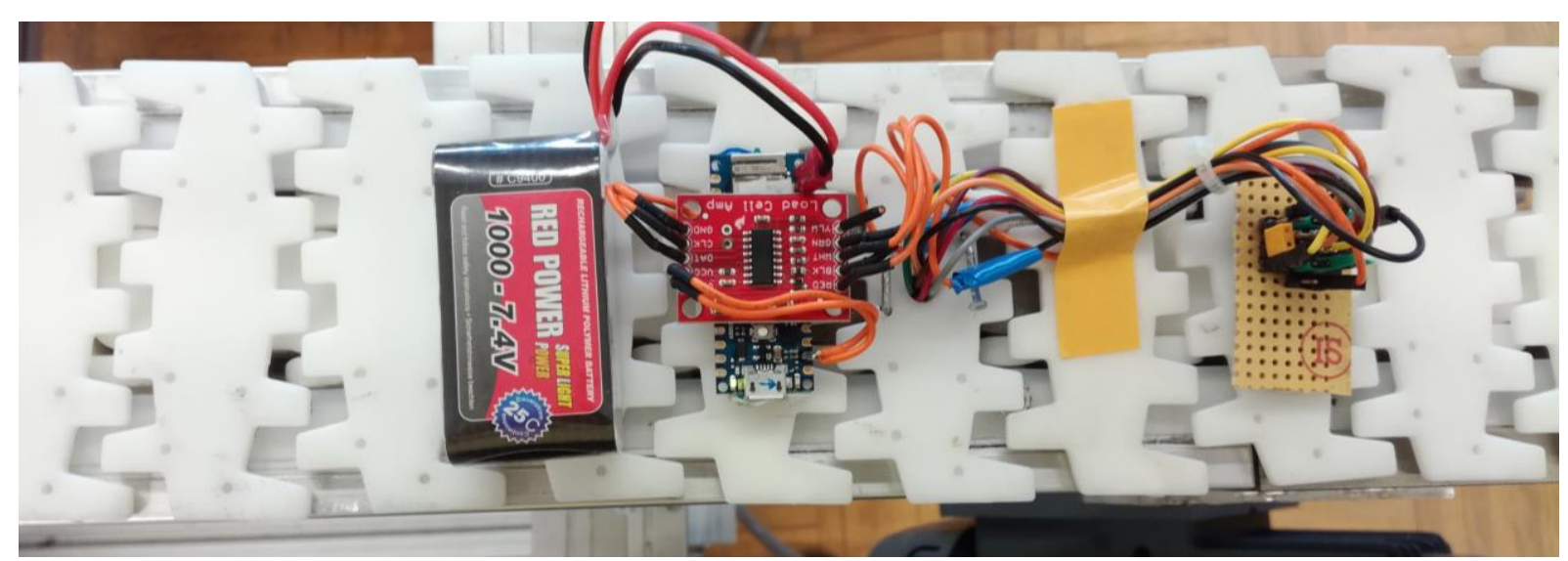

Figure 3: Prototype of the measuring chain section 


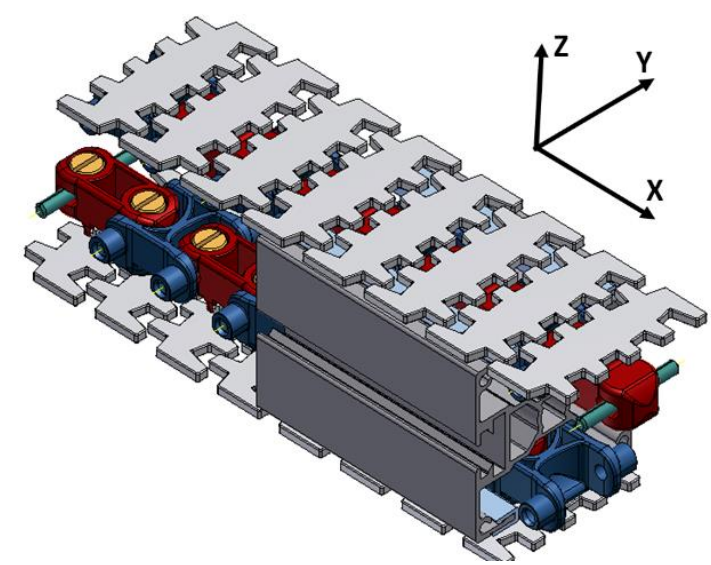

Figure 4: Coordinate system of the inertial sensor relative to the chain

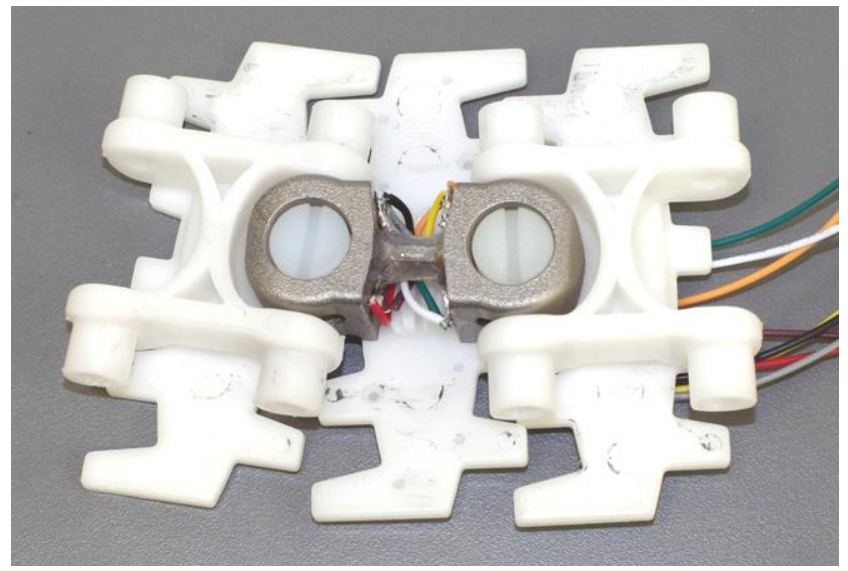

Figure 5: Metal chain link with applied strain gauges

However, the main dimensions of the selected components as well as the possibility to further reduce the size of the circuit boards will allow a complete integration of the electronics into the chain links in the future, so that the outer dimensions of the chain are not changed according to the target position and thus the conveyor function is not affected.

The following sections describe the components of the system in more detail:

\section{Inertial Measurement Unit (IMU)}

With the 6-axis IMU located on the main board, acceleration in $\mathrm{X}, \mathrm{Y}$ and $\mathrm{Z}$ directions (see Figure 4) and the corresponding rotation rates can be measured at a sampling rate of $100 \mathrm{~Hz}$. In addition to the particularly longitudinal chain oscillations, the exact position of the chain link can also be captured, so that a determination of position or tracking of the corresponding link within the conveyor system is possible without the need for additional sensors.

When the measuring chain section is moved through the conveyor, the sign of the $\mathrm{Z}$ acceleration value is used to identify whether the measuring chain is on the lower or upper run. A movement through horizontal curves can be detected with the rotation rate around the Z-axis. The combination of several sensor values is necessary for the detection of the drive unit, the redirection unit and sloped sections. The sensor values can thus be used to reliably determine in which section type the measuring chain is located. The exact position in the respective section is then determined with the aid of evaluation software in which the layout is stored. The prerequisite for exact tracking is a quasi-constant conveying speed.

\section{Tractive Force Sensor}

The measurement of the tractive force in the chain is currently carried out via a strain gauge, which is a great challenge. Since positioning directly on the plastic chain has proven to be unfavourable due to the visco-elastic material properties and the resulting drift of the measured value, only metallic parts are considered. In first preliminary tests, two variants have been considered so far. In the first one, the strain gauges are placed on the stainless steel pin, which connect two chain links. In the second one, the chain link is replaced by a metal body produced by additive manufacturing (see Figure 5). An important requirement 
is that the chain link is not damaged too much by the strain gauge and that the measuring point of the strain gauge shows a force-strain behaviour that is as linear as possible. Further investigations are necessary for this purpose.

\section{Energy Supply}

Depending on the size, Li-ion rechargeable batteries can supply the measuring system with energy for a few hours up to a whole day. A DC/DC converter is used to provide a constant board voltage independent of the discharge characteristics of the accumulators.

A permanent monitoring of the battery voltage should prevent the degradation of the batteries by deep discharge. If the voltage falls below a critical level, the system switches off. This increases the durability of the batteries and enables the system to be used for longer periods.

The battery life depends on how much data has to be recorded and sent per time. In order to save power and increase the runtime, it would be possible in the future, to query only those accelerations of the IMU sensor that are required for evaluation. Furthermore, the sampling rate could be optimised or the measuring electronics could be set to a temporary stand-by mode in less interesting sections of the conveyor, e.g. in the lower run.

\section{Data Transmission}

The data transfer is done via a Wi-Fi module located on the board. It transmits in the $2.4 \mathrm{GHz}$ band and supports the $802.11 \mathrm{~b} / \mathrm{g} / \mathrm{n}$ standards. The maximum data rate of the transmitter is a multiple of what is required to transmit the measurement data. The transmission protocol is TCP/IP based, so that the measurement chain section gets its own IP address. This would make it possible in future to use several measuring chain sections in a conveyor chain, in which each can be addressed via its own IP address. The board also contains a buffer memory (ring buffer), in which the measurement data is temporarily stored before being sent to the evaluation station. In this way disturbances and interruptions on the transmission line can be compensated.

\section{Analysis and Evaluation Software}

An essential component of the overall system is the specially developed analysis and evaluation software, which processes the measurement data collected by the circulating measuring chain section and transferred to a PC via Wi-Fi. This has the following tasks:

- Operation/control of the measuring chain section.

- Establishment and upkeep of the radio connection to the measuring chain.

- Fetch of the data from the measuring chain.

- Evaluation and graphic representation of the measured data.

- Position determination (tracking) of the measuring chain section in the conveyor system. 


\section{Test Results}

A sliding chain conveyor system was used for experimental studies with the measuring chain. The conveyor is built up of three straight sections and two 90-degree curves, which are realised by curved wheels (see Figure 6). The accelerations were quantified and analysed in initial measurements. The measurement of tractive forces has not yet been implemented.

Figure 7 shows the curves of the accelerations in $\mathrm{X}$ - and Z-direction as well as the rotation rate RZ. The diagram represents one circulation. All curves illustrate typical vibrations for chain conveyor systems. The X-direction (longitudinal) generally reveal relatively large amplitudes. In the Z-direction (vertical) the basic oscillations are lower, but here several clear peaks can be seen, which indicate irregularities such as impacts at transition points between slide rails or when the chain is re-enter the guiding system after curved wheels or redirections. The Z-values also reveal the change from the lower to the upper run in the chain redirection after approx. $45 \mathrm{~s}$ by changing the leading sign. The measured value of the rotation rate RZ (blue curve) can be used to reliably detect horizontal curves in the chain conveyor.

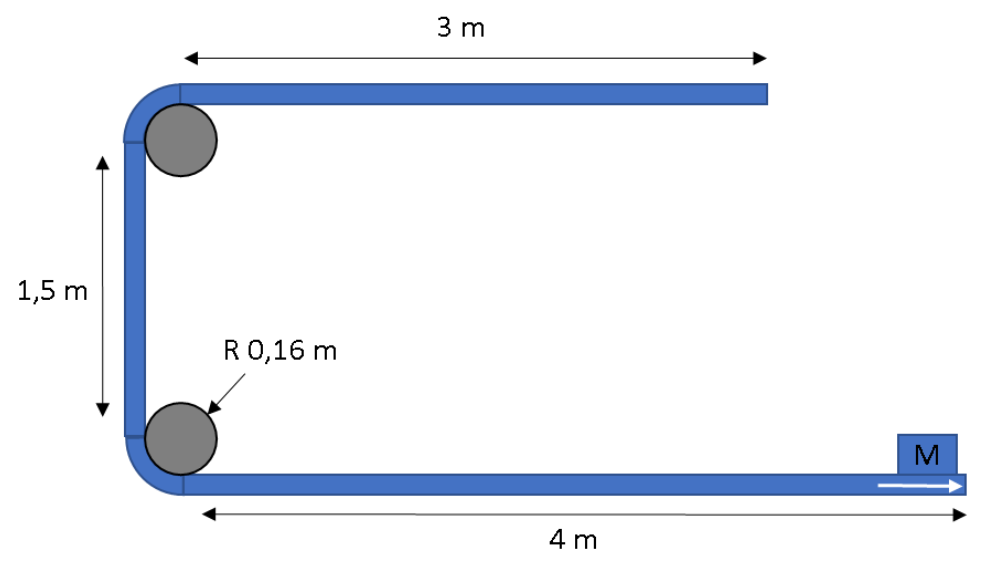

Figure 6: Layout of the conveyor way used

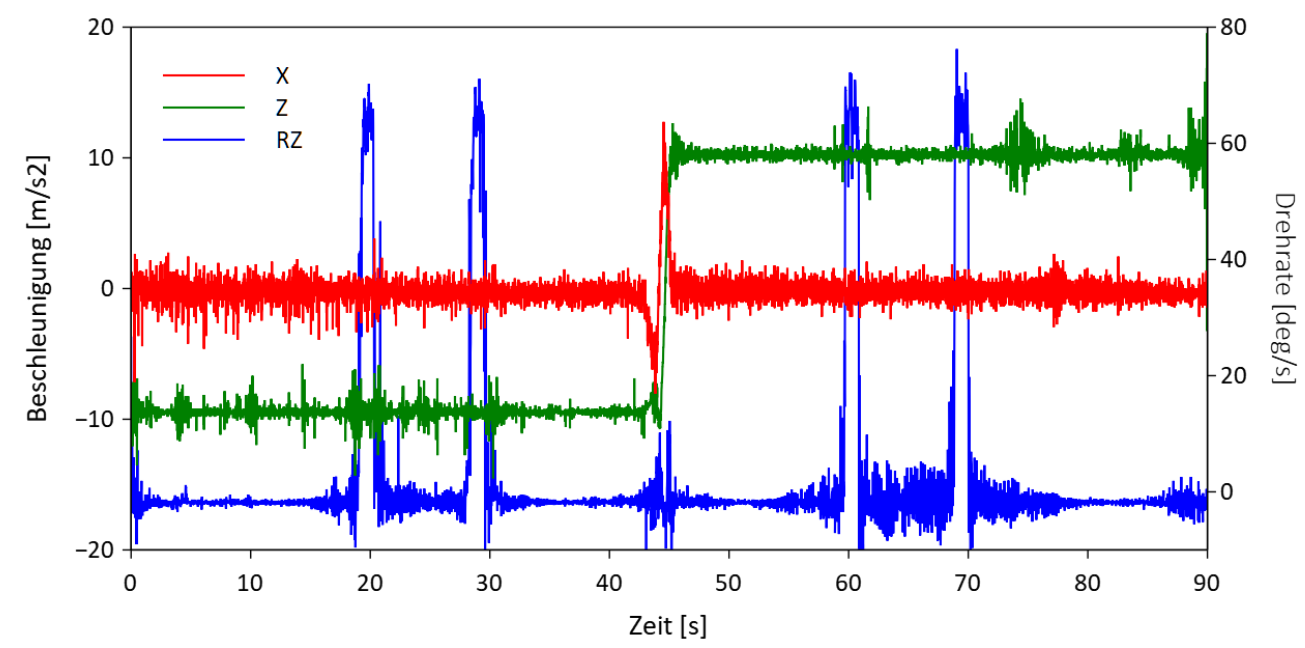

Figure 7:

Measured acceleration in $\mathrm{X}$ and $\mathrm{Z}$ direction and the rotation rate around the $\mathrm{Z}$ axis for one chain rotation 


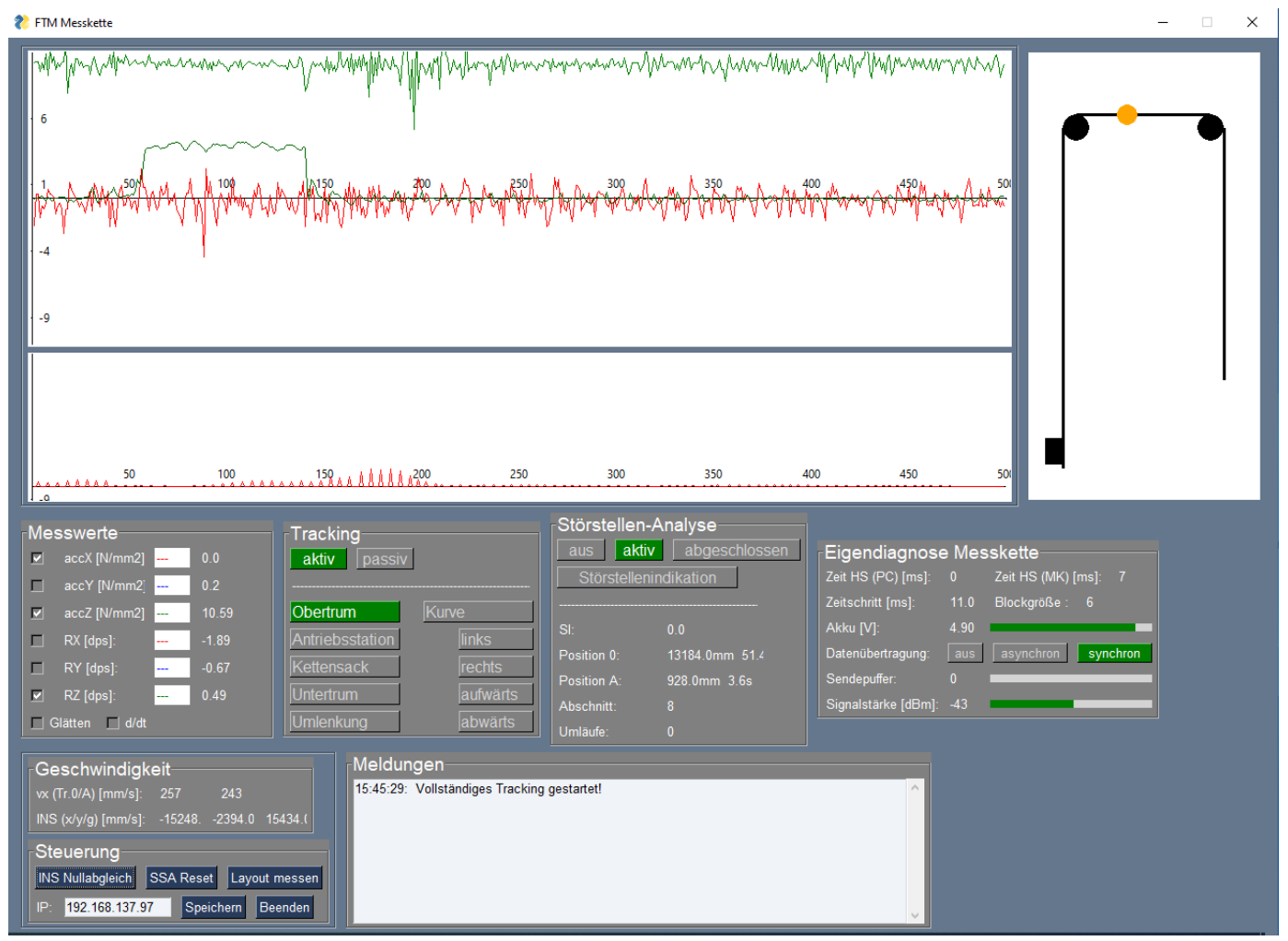

Figure 8: Screenshot of the analysis and evaluation software. Live measured data is shown as curve plots in upper left box and the position of measuring chain section is shown as an orange dot in the upper right box.

Figure 8 shows a screenshot of the analysis and evaluation software, which controls the recording of measured values and displays them in the live view almost in real-time. The current position of the measuring chain in the conveyor system is marked by a coloured dot. Furthermore, a self-diagnosis is carried out regarding the battery voltage, the signal reception quality and the sampling rate. In the future, more tools can be integrated in the software.

\section{Potential Application}

The measurement chain opens up a wide range of applications, which can be generated by suitable analysis of the provided data. Some of these possibilities are briefly presented hereinafter.

\section{General Analysis Functions}

A detailed analysis of chain conveyor systems is possible with the measuring chain. This includes on the one hand the comparison with the calculation bases available so far and those to be further developed for the determination of the chain tractive force curve and the chain vibrations, by which the accuracy of the plant dimensioning can be improved. This is particularly important for complex systems with many deflections and/or differently loaded sections, where the measured drive torque alone cannot provide sufficient information. 
On the other hand, the measuring chain section can be used for system analysis of running systems. Thus, for example, in multi-track conveyor systems with a shared drive shaft, the exact load distribution on the individual chain tracks can be determined.

In addition, the maximum tractive force of the chain does not necessarily have to be applied to the drive station in all chain conveyors. Under certain load conditions local force increases can occur due to vibrations which clearly exceed the average tractive force at the drive.

\section{Friction Mapping}

It is known that the magnitude of the coefficients of friction depends on many parameters such as speed, normal force, running-in or wear behaviour of the chains and slide rails. [12-14] In contrast, current calculation bases for chain conveyor systems usually only take into account a constant coefficient of friction that is valid for the whole system. With the aid of the measuring chain and the tracking functionality, local differences in the coefficient of friction can be detected in the conveyor layout by calculating the tractive forces of the chain with a known load (normal force) section by section. In this way, important findings can be obtained, which contribute to the improvement of dimensioning methods.

\section{Detection of Discontinuity}

Areas of the conveyor layout with disturbances, such as poorly mounted slide rails, can be detected in connection with the tracking. For this purpose, a disturbance indicator can be calculated from the combination of the acceleration values and the tractive force. In order to achieve a high reliability of the detection, additional test series are necessary.

\section{Monitoring of Chain Conveyors / Failure Prediction}

In the future, a permanent monitoring of chain conveyor systems, especially in failurecritical applications, is also conceivable. The measuring sections integrated in the chains could detect disturbances or overloads at the earliest possible time and transmit them to the plant operator so that serious damage or a plant failure can be avoided as far as possible. In addition, a lifetime prognosis or failure prediction for the sliding chain could be realised by using the tractive force of the chain for damage accumulation.

\section{Conclusion and Outlook}

The paper described the development of a measurement chain section that can be integrated into a conveyor chain. In the circulating chain, the tractive force as well as the accelerations in 6 axes can be measured and the circulations recorded. The data is continuously transmitted by radio to an evaluation station. A Software runs on this station which analyses the received data according to various criteria and also provides tracking functionality.

In initial functional tests, which form the basis for further work, measurement data could be gathered and successfully evaluated. This happened contact-free and almost in real time. In the future, the circuit board with the measuring electronics is to be made even more compact in order to achieve complete integration into the chain without influencing the flow of the 
conveyed goods. In this context, further investigations are also necessary for the suitable application of the strain gauges on the chain body in order to be able to measure tractive forces reliably and permanently. A further important objective is the control and evaluation software, with the help of which the measuring chain can be extended by new application possibilities.

This work contributes to increasing the reliability and service life of conveyor systems with plastic chains by means of optimised dimensioning and target-oriented system monitoring.

\section{References}

[1] Bosch Rexroth AG: Kettenfördersystem VarioFlow plus (Produktkatalog), Version 2.0.

[2] FlexLink: Conveyor chain guide (2015), www.flexlink.com (Accessed on 26.09.2020)

[3] Habasit AG: HabaCHAIN ${ }^{\circledR}$ Slat and Conveyor Chains Product Guide. www.habasit.com (Accessed on 26.09.2020)

[4] Ammeraal Beltech Modular A/s: Engineering Manual. www.unichains.com (Accessed on 26.09.2020)

[5] Ammeraal Beltech Modular A/S: Innovative belt and chain solutions for every industry \& application. www.unichains.com (Accessed on 26.09.2020)

[6] Movex S.p.A.: Product Catalog (2019), www.movexii.com (Accessed on 26.09.2020)

[7] Rexnord: FlatTop Engineering Manual (2016), www.rexnord.com (Accessed on 26.09.2020)

[8] Rexnord: Smart Condition Monitoring System (2020). www.rexnord.com (Accessed on 26.09.2020)

[9] Auerbach, P.: Zur Beanspruchung und Lebensdauer raumgängiger Gleitketten aus Kunststoff. TU Chemnitz, Dissertation, 2006. https://nbn-resolving.org/urn:nbn:de:swb:ch1-200600396

[10] Bartsch, R.: Erweiterung der Dimensionierungsgrundlagen für Gleitkettenfördersysteme. TU Chemnitz, Dissertation, 2017. https://nbn-resolving.org/urn:nbn:de:bsz:ch1-qucosa-229404

[11] Sumpf, J., Bankwitz, H., Nendel, K., Rasch F.: Novel calculation method for chain conveyor systems. Logistics Journal: referierte Veröffentlichungen, Vol. 2014. http://dx.doi.org/10.2195/lj_Rev_sumpf_en_201411_01

[12] Erhard, G.: Konstruieren mit Kunststoffen. 4. Auflage. Carl Hanser Verlag, München, 2008

[13] Czichos, H., Habig, K-H. (Hrsg.): Tribologie-Handbuch. Tribometrie, Tribomaterialien, Tribotechnik. 5. Auflage, 2020, ISBN 978-3-658-29484-7 (eBook)

[14] Bartsch, R.; Sumpf, J.; Bergmann, A.: Grenzbelastung von thermoplastischen Gleitkontakten am Beispiel von POM - PE Paarungen. Tribologie + Schmierungstechnik, Jg. 65 (2018), H. 2/2018, S. 62-68. ISSN 0724-3472.

[15] Strobel, J.: Untersuchung von Schwingungen an einem Stetigfördersystem mit Kunststoffgleitketten, TU Chemnitz, Dissertation, 2018. https://nbn-resolving.org/urn:nbn:de:bsz:ch1-qucosa2-211447

[16] https://www.boschrexroth.com/de/de/produkte/produktgruppen/montagetechnik/news/kettenfoerder systeme-varioflow-plus/index (Accessed on 26.09.2020)

[17] https://blog.krones.com/bewusst-nachhaltig/201609ko06_0086_korr_preview_layout/ (Accessed on 26.09.2020)

[20] Dutschk, V.: Oberflächenkräfte und ihr Beitrag zu Adhäsion und Haftung in glasfaserverstärkten Thermoplasten. Dissertation, Dresden (2000)

[21] Prokopovich, P., et al.: Comparison of JKR- and DMT-based multi-asperity adhesion model - theory and experiment. Colloids and Surfaces A: Physicochem. Eng. Aspects 383: 95-101 (2011)

[22] Bergström, J.: Mechanics of Solid Polymers: Theory and Computational Modeling, Elsevier (2015)

[23] https://polymerfem.com/three-network-model/ (Accessed on 07.10.2020) 